\title{
Das SCF-LCGO-Verfahren. IV. Atomrechnungen (Schluß)
}

\author{
H. Preuss \\ Max-Planck-Institut für Physik und Astrophysik, München \\ (Z. Naturforschg. 20 a, 1290-1298 [1965]; eingegangen am 10. Juni 1965)
}

\begin{abstract}
Es werden die Gesamtenergien der Atome und Ionen für folgende Konfigurationen und Kernladungen berechnet:$$
(1 \mathrm{~s})^{2} \quad 2 \leqq Z \leqq 10 ; \quad(1 \mathrm{~s})^{2}(2 \mathrm{~s})^{2}(2 \mathrm{p})^{n} \quad 4+n \leqq Z \leqq 10 \quad(n=0,2,4,6)
$$

und die Frage geklärt, wieviel Gauss-Funktionen notwendig sind, damit eine ausreichende Genauig. keit erreicht werden kann. Die Ergebnisse werden in Form von Tabellen angegeben, sowie auch die dazugehörigen Lagen der Gauss-Funktionen im Raum und deren $\eta$-Werte. Es genügt, für (ls) ${ }^{2}$ vier Funktionen, für $(1 \mathrm{~s})^{2}(2 \mathrm{~s})^{2}(2 \mathrm{p})^{n} 5+2 n$ Funktionen zu verwenden. Die Abweichungen der Gesamtenergie im Vergleich zu den bekannten HF-Werten liegen in allen Fällen zwischen 2\% und $1 \%$. Die Zusammenhänge der verwendeten Parameter mit $Z$ im Energieminimum lassen sich teilweise durch einfache Funktionen sehr gut approximieren. Bei der Diskussion der Ergebnisse wird besonders auf die jetzt möglichen Molekülrechnungen mit dem SCF-LCGO-Verfahren eingegangen.
\end{abstract}

Damit das SCF-LCGO-Verfahren ${ }^{1}$ auf Moleküle angewendet werden kann, die Atome mit einer Kernladung $Z \geqq 2$ enthalten, ist es notwendig, Rechnungen anzustellen, die Hinweise geben, wieviele GaussFunktionen notwendig sind, um die Dichteverteilungen der Elektronen in Atomen und Atomrümpfen ausreichend genau darzustellen. Da es sich dabei um ein SCF-Verfahren handelt, kann damit nur die energieminimisierende Dichteverteilung in diesem Rahmen erhalten werden. Die Frage nach der Anzahl der Gauss-Funktionen

$$
\begin{aligned}
\varphi_{\lambda}\left(\mathfrak{r}_{\lambda} ; \eta_{\lambda}\right) & \equiv \varphi_{\lambda}\left(x_{\lambda}, y_{\lambda}, z_{\lambda} ; \eta_{\lambda}\right) \\
& =\left(2 \eta_{\lambda} / \pi\right)^{3 / 4} \exp \left\{-\eta_{\lambda}\left(\mathfrak{r}-\mathfrak{r}_{\lambda}\right)^{2}\right\}
\end{aligned}
$$

im LCGO-Ansatz führt zu der Frage, welche $\eta_{\lambda}$ - und $\mathfrak{r}_{\lambda}$-Werte gewählt werden müssen, damit die Konfigurationen $(1 \mathrm{~s})^{2} ;(1 \mathrm{~s})^{2}(2 \mathrm{~s})^{2}$ und $(1 \mathrm{~s})^{2}(2 \mathrm{~s})^{2}(2 \mathrm{p})^{n}$ $(n=2,4,6)$ mit nahezu HF-Genauigkeit erfaßt werden.

Im SCF-LCAO-Verfahren werden als Atomfunktionen die Slater-Funktionen $\Phi$ verwendet

$$
\begin{aligned}
& \Phi_{n, l, m}=\frac{(2 \alpha)^{n^{\prime}+1 / 2}}{\left[\pi\left(2 n^{\prime}\right) !\right]^{1 / 2}}\left[\frac{(2 l+1)(l+|m|) !}{2(l-|m|) !}\right]^{1 / 2} \\
& \cdot r^{n^{\prime}-1} e^{-a r} P_{l}^{|m|}(\cos \vartheta)\left\{\begin{array}{lll}
1 / \sqrt{2} & \text { für } & m=0 \\
\cos (m \varphi) & & \\
\sin (m \varphi) & \text { für } & |m|>0,
\end{array}\right.
\end{aligned}
$$

deren Parameter $n^{\prime}$ und $\alpha$ aus dem sogenannten „SLATER-Rezept“ erhalten werden, welches einen Zusammenhang zwischen $n^{\prime}$ und $\alpha$ und der Anzahl der Elektronen im Atom und deren Einteilchenzustände

1 H. Preuss, Z. Naturforschg. 19 a, 1335 [1964] (Teil I), 20 a, 17 [1965] (Teil II), 20 a, 21 [1965] (Teil III). $(n, l, m)$ herstellt, wobei

$$
\alpha=(Z-\sigma) / n^{\prime},
$$

und in $\sigma$ das obige Rezept Anwendung findet ${ }^{2}$. Die Slater-Funktionen selbst sind Eigenfunktionen der SchröDINGER-Gleichung

$$
\begin{aligned}
\left\{\frac{1}{2} \Delta-\frac{n^{\prime} \alpha}{r}\right. & \left.+\frac{1}{2} \frac{n(n-1)-l(l+1)}{r^{2}}\right\} \Phi_{n, l, m} \\
& =-\frac{1}{2} \alpha^{2} \Phi_{n, l, m},
\end{aligned}
$$

wenn $l$ die Drehimpulsquantenzahl des jeweiligen Elektrons bedeutet. Da wir uns hier vorerst nur auf die Elektronen der K- und L-Schale beschränken wollen, so erfüllen im einzelnen die $1 \mathrm{~s}$, $2 \mathrm{~s}$ - und $2 \mathrm{p}$ Slater-Funktionen die folgenden Differentialgleichungen (in atomaren Einheiten) :

$$
\begin{aligned}
& \text { ls: }\left[\frac{1}{2} \Delta-\frac{Z-\sigma_{1 \mathrm{~s}}}{r}\right] \Phi_{1,0,0}= \\
& -\frac{1}{2}\left[Z-\sigma_{1 \mathrm{~s}}\right]^{3} \Phi_{1,0,0}, \\
& 2 \mathrm{~s}:\left[\frac{1}{2} \Delta-\frac{Z-\sigma_{2 \mathrm{~s}}}{r}+\frac{1}{r^{2}}\right] \Phi_{2,0,0}= \\
& -\frac{1}{2}\left[\frac{Z-\sigma_{2 \mathrm{~s}}}{2}\right]^{2} \Phi_{2,0,0}, \\
& 2 \mathrm{p}:\left[\frac{1}{2} \Delta-\frac{Z-\sigma_{2 \mathrm{p}}}{r}\right] \Phi_{2,1,0}= \\
& -\frac{1}{2}\left[\frac{Z-\sigma_{2 p} \mathrm{p}}{2}\right]^{2} \Phi_{2,1,0}
\end{aligned}
$$

(ebenfalls für $\Phi_{2,1,1}$ und $\Phi_{2,1,-1}$ ).

Die Gln. (4a) und (4 c) stellen die SchrödingerGleichungen eines wasserstoffähnlichen Systems dar,

2 J. C. Stater, Phys. Rev. 36, 57 [1930]. 
wenn die Kernladung $Z-\sigma$ beträgt. Nach ${ }^{3-5}$ ist dieses Einelektronensystem schon mit Gauss-Funk-

tionen berechnet worden. Bezeichnen wir die Anzahl der Funktionen (1) im LCGO-Ansatz mit $M$, so liegen folgende Ergebnisse vor ${ }^{3-5}$ :

$$
\begin{aligned}
& \varepsilon=-0,42441, \\
& \varepsilon=-0,4858,
\end{aligned}
$$

$$
\varepsilon=-0,4970 \text {, }
$$

$$
\begin{array}{r}
M=4, \frac{1}{11,262}\left[\varphi\left(0 ; 2,8399(Z-\sigma)^{2}\right)+4,90 \varphi\left(0 ; 0,5789(Z-\sigma)^{2}\right)\right. \\
\left.\quad+6,70 \varphi\left(0 ; 0,1395(Z-\sigma)^{2}\right)+0,156 \varphi\left(0 ; 17,499(Z-\sigma)^{2}\right)\right] ; \varepsilon=-0,4990 \quad\left(\varepsilon_{\text {exakt }}=-0,5\right),
\end{array}
$$$$
M=2,2 \mathrm{p}: \quad \frac{1}{0,3921}\left[\varphi\left(\frac{0,933}{Z-\sigma}, 0,0 ; 0,0460(Z-\sigma)^{2}\right)-\varphi\left(-\frac{0,933}{Z-\sigma} 0,0 ; 0,0460(Z-\sigma)^{2}\right)\right]
$$

$$
\varepsilon=-0,1132 \text {, }
$$

$$
M=4, \frac{1}{2,1644}\left[\varphi\left(\frac{0,80}{Z-\sigma}, 0,0 ; 0,1687(Z-\sigma)^{2}\right)+6,1483 \varphi\left(\frac{0,80}{Z-\sigma}, 0,0 ; 0,0337(Z-\sigma)^{2}\right)\right.
$$

$$
\left.-\varphi\left(-\frac{0,80}{Z-\sigma}, 0,0 ; 0,1687(Z-\sigma)^{2}\right)-6,1483 \varphi\left(-\frac{0,80}{Z-\sigma}, 0,0 ; 0,0337(Z-\sigma)^{2}\right)\right] ; \varepsilon=-0,1231,
$$

$M=6, \frac{1}{3,5956}\left[\left(\varphi \frac{0,362}{Z-\sigma}, 0,0 ; 0,3057(Z-\sigma)^{2}\right)+9,0 \varphi\left(\frac{0,362}{Z-\sigma}, 0,0 ; 0,0734(Z-\sigma)^{2}\right)\right.$

$$
\begin{array}{r}
+17,3 \varphi\left(\frac{0,362}{Z-\sigma}, 0,0 ; 0,0244(Z-\sigma)^{2}\right)-\varphi\left(\frac{0,362}{Z-\sigma}, 0,0 ; 0,3057(Z-\sigma)^{2}\right) \\
\left.-9,0 \varphi\left(-\frac{0,362}{Z-\sigma}, 0,0 ; 0,0734(Z-\sigma)^{2}\right)-17,3 \varphi\left(-\frac{0,362}{Z-\sigma}, 0,0 ; 0,0244(Z-\sigma)^{2}\right)\right] \\
\quad \mathcal{E}=-0,1247 \quad\left(\varepsilon_{\text {exakt }}=-0,125\right) .
\end{array}
$$

Bezüglich der Angaben von ${ }^{3}$ und ${ }^{4}$ bestehen in einigen Fällen sehr kleine Abweichungen, die vermutlich darauf zurückzuführen sind, daß das Energieminimum sehr flach ist.

Die 2s-Zustände des H-Atoms sind ebenfalls berechnet worden ${ }^{3-5}$, da aber in $(4 \mathrm{~b})$ noch ein Glied $1 / r^{2}$ auftritt, welches im Vielelektronensystem als effektives Potential gedeutet werden kann, so wollen wir hier darauf nicht eingehen. Im Falle des $2 \mathrm{~s}-\mathrm{Zu}$ standes im Vielelektronenatom [Gl. (4 b)] besteht die Aufgabe darin, Näherungen für die Lösungen der Gl. (4 b) zu finden.

Wie schon im Teil III $\left(\mathrm{H}_{3}{ }^{+}\right.$-Molekül), wollen wir die Verhältnisse dadurch anschaulich machen, daß wir jeder Gauss-Funktion einen Kreis zuordnen. Danach entsprechen die Funktionen $(5 \mathrm{a})-(5 \mathrm{~d})$ der Abb. 1.
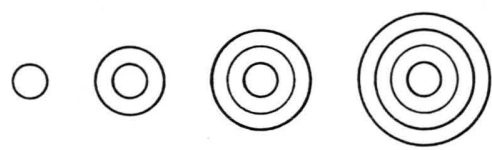

Abb. 1 .
Für die Gln. (6a) - (6c) zeichnen wir Abb. 2.

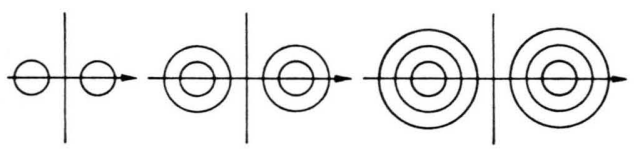

Abb. 2.

Im Falle der 2p-Funktionen ergeben sich die anderen beiden Funktionen $\left(2 \mathrm{p}_{x}, 2 \mathrm{p}_{y}\right)$, indem ihre Position entsprechend auf die $x$ - oder $y$-Achse verlegt wird.

Das einfachste Beispiel einer $\mathrm{d}_{x y}$-Funktion könnte dann durch Abb. 3 dargestellt werden, doch wollen wir vorerst darauf nicht näher eingehen.

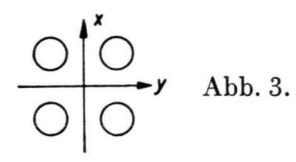

5 H. Preuss, Integraltafeln zur Quantenchemie, Band IV, Springer-Verlag, Berlin 1960. 


\section{Der Gang der Rechnung und die Ergebnisse}

Um gute Energien der SCF-Atomrechnungen zu erhalten, ist es notwendig, daß besonders die inneren Elektronen $(1 \mathrm{~s})^{2}$ so gut wie möglich in ihrer Dichteverteilung erfaßt werden. Es ist daher zuerst die (ls $)^{2}$-Konfiguration der Atome und Ionen für $2 \leqq Z \leqq 10$ mit $M=4$ nach $(5 \mathrm{~d})$ berechnet worden, dabei setzen wir

$$
\eta_{j}^{\prime}=\eta_{j}^{(\mathrm{H})}(Z-\sigma)^{2},
$$

wobei $\eta^{\prime}$ die $\eta$-Werte des freien H-Atoms nach $(5 \mathrm{~d})$ bedeuten.

Für jedes $Z$ wurde $\sigma$ auf Energieminimum variiert. Die Tab. 1 gibt die $\sigma$-Werte sowie einen Vergleich mit den zur Zeit besten SCF-Werten ${ }^{6}$ der Gesamtenergie wieder. Für $\eta^{(\mathrm{H})}$ wurde nach $(5 \mathrm{~d})$ gesetzt:

$$
\begin{array}{ll}
\eta_{1}{ }^{(\mathrm{H})}=0,1395 ; & \eta_{3}{ }^{(\mathrm{H})}=2,8399 ; \\
\eta_{2}{ }^{(\mathrm{H})}=0,5789 ; & \eta_{4}{ }^{(\mathrm{H})}=17,4990 .
\end{array}
$$

\begin{tabular}{|c|c|c|c|c|c|c|}
\hline$Z$ & $\frac{\text { Atom }}{\text { Ion }}$ & $\sigma$ & $-\varepsilon_{\min }$ & $\begin{array}{c}-\varepsilon_{\mathrm{SCF}} \\
\operatorname{nach}(6)\end{array}$ & $\Delta \varepsilon$ & $\%$ \\
\hline 2 & $\mathrm{He}$ & 0,5399 & 2,8548 & 2,8617 & 0,0069 & 0,24 \\
\hline 3 & $\mathrm{Li}^{+}$ & 0,5847 & 7,2214 & 7,2364 & 0,0150 & 0,21 \\
\hline 4 & $\mathrm{Be}^{2+}$ & 0,6246 & 13,5844 & 13,6113 & 0,0269 & 0,20 \\
\hline 5 & $\mathrm{~B}^{3+}$ & 0,6603 & 21,9438 & 21,9862 & 0,0424 & 0,19 \\
\hline 6 & $\mathrm{C}^{4+}$ & 0,6924 & 32,2994 & 32,3612 & 0,0618 & 0,19 \\
\hline 7 & $\mathrm{~N}^{5+}$ & 0,7214 & 44,6514 & 44,7362 & 0,0848 & 0,19 \\
\hline 8 & $\mathrm{O}^{6+}$ & 0,7478 & 58,9998 & 59,1111 & 0,1113 & 0,19 \\
\hline 9 & $\mathrm{~F}^{7+}$ & 0,7719 & 75,344 & 75,4861 & 0,1417 & 0,19 \\
\hline 10 & $\mathrm{Ne}^{8+}$ & 0,7940 & 93,6853 & 93,1758 & 0,1758 & 0,19 \\
\hline
\end{tabular}

Tab. 1.

Die folgende Tab. 2 gibt die zum Energieminimum gehörenden Koeffizienten $C_{j k}$ wieder, wobei

$$
\begin{aligned}
\psi_{1}= & C_{11} \varphi_{1}\left(\eta_{1}^{\prime}\right)+C_{12} \varphi_{2}\left(\eta_{2}{ }^{\prime}\right)+C_{13} \varphi_{3}\left(\eta_{3}{ }^{\prime}\right) \\
& +C_{14} \varphi_{4}\left(\eta_{4}^{\prime}\right)
\end{aligned}
$$

und die Einelektronenfunktion $\psi_{1}$ normiert ist.

\begin{tabular}{|r|c|c|c|c|}
\hline$Z$ & $C_{11}$ & $C_{12}$ & $C_{13}$ & $C_{14}$ \\
\hline 3 & 0,524 & 0,478 & 0,131 & 0,019 \\
4 & 0,534 & 0,474 & 0,123 & 0,018 \\
5 & 0,540 & 0,471 & 0,118 & 0,017 \\
6 & 0,545 & 0,469 & 0,114 & 0,016 \\
7 & 0,549 & 0,467 & 0,112 & 0,016 \\
8 & 0,552 & 0,465 & 0,112 & 0,016 \\
9 & 0,554 & 0,463 & 0,109 & 0,015 \\
10 & 0,557 & 0,462 & 0,107 & 0,015 \\
\hline
\end{tabular}

Tab. 2.

Der erste Index in $C_{j k}$ gibt die Numerierung der einzelnen Einelektronenzustände wieder. $j=1$ be-

${ }^{6}$ C. C. Roothana, L. M. Sachs u. A. W. Weiss, Rev. Mod. Phys. 32, 186 [1960]. deutet, daß diese $C_{j k}$ zum tiefsten Zustand (1s) gehören.

Die Rechnungen haben ergeben, daß die Funktion $\sigma=\sigma(Z)$ für $\varepsilon_{\min }$ sehr gut durch

$$
\sigma(Z)=\frac{0,431+0,093 Z}{1+0,714 Z} ; \quad(2 \leqq Z \leqq 10)
$$

dargestellt werden kann. Die Abhängigkeit der Energie von $\sigma$ ist im Minimum von $\mathcal{E}$ ziemlich schwach (flaches Minimum), so daß die Funktion (10) für die üblichen Genauigkeitsforderungen völlig ausreicht.

Um die $(1 s)^{2}(2 s)^{2}$-Konfiguration ohne eine $z u$ große Anzahl von Gauss-Funktionen berechnen zu können, wurden die vier $\eta$ nach (8) beibehalten und nur noch eine Gauss-Funktion $\varphi_{5}$ hinzugenommen. Wir haben also, in Erweiterung von (7) und (8),

$\eta_{1}{ }^{\prime}=0,1395\left(Z-\sigma_{1}\right)^{2}, \quad \eta_{4}{ }^{\prime}=17,4990\left(Z-\sigma_{1}\right)^{2}$,

$\eta_{2}^{\prime}=0,5789\left(Z-\sigma_{1}\right)^{2}, \quad \eta_{5}{ }^{\prime}=0,016\left(Z-\sigma_{2}\right)^{2}$,

\begin{tabular}{|c|c|c|c|c|c|c|c|}
\hline$Z$ & $\begin{array}{l}\text { Atom/ } \\
\text { Ion }\end{array}$ & $\sigma_{1}$ & $\sigma_{2}$ & $-\varepsilon_{\min }$ & $\begin{array}{c}-\varepsilon_{\mathrm{SCF}} \\
\operatorname{nach}(6)\end{array}$ & $\Delta \varepsilon$ & $\%$ \\
\hline 4 & $\mathrm{Be}$ & 4375 & 1,4460 & 14,5 & 14 & 0,0359 & 0,25 \\
\hline 5 & $\mathrm{~B}^{+}$ & 0 & 1,2600 & 24, & & 53 & 0,23 \\
\hline 6 & $\mathrm{C}^{2}$ & 0,3750 & 1,0740 & 36,3285 & 36 , & 0,0800 & 0,22 \\
\hline 7 & $\mathrm{~N}^{3+}$ & 0,3571 & 0,8889 & 50,9722 & 51 , & 0,1101 & 0,22 \\
\hline 8 & $\mathrm{O}^{4+}$ & 0,3438 & 0,7020 & 68,1119 & 68,2577 & 0,1458 & 0,21 \\
\hline 9 & $\mathrm{~F}^{5+}$ & 0,3333 & 0,5160 & 87,7469 & 87,9341 & 0,1872 & 0,21 \\
\hline 10 & $\mathrm{Ne}^{6+}$ & 0,3250 & 0,3333 & 109,8767 & 110,1110 & 0,2343 & 0,21 \\
\hline
\end{tabular}

$\eta_{3}{ }^{\prime}=2,8399\left(Z-\sigma_{1}\right)^{2}$,

und somit zwei $\sigma$ eingeführt, die beide bezüglich Energieminimum variiert wurden. Die folgenden Tab. 3 und 4 geben die Ergebnisse wieder.

Tab. 3.

\begin{tabular}{|r|c|c|c|c|c|}
\hline$Z$ & $-C_{21}$ & $-C_{22}$ & $-C_{23}$ & $-C_{24}$ & $C_{25}$ \\
\hline 4 & 0,180 & 0,092 & 0,020 & 0,003 & 1,034 \\
5 & 0,216 & 0,113 & 0,022 & 0,003 & 1,049 \\
6 & 0,240 & 0,127 & 0,024 & 0,004 & 1,060 \\
7 & 0,257 & 0,137 & 0,025 & 0,004 & 1,069 \\
8 & 0,269 & 0,145 & 0,026 & 0,004 & 1,076 \\
9 & 0,279 & 0,151 & 0,027 & 0,004 & 1,082 \\
10 & 0,287 & 0,156 & 0,028 & 0,004 & 1,087 \\
\hline
\end{tabular}

Tab. 4.

Die Zusammenhänge der $\sigma_{1}$ und $\sigma_{2}$ mit $Z$ können wieder sehr gut durch

$$
\begin{aligned}
\sigma_{1}(Z)= & 0,25(1+3 / Z), \\
& (4 \leqq Z \leqq 10) \\
\sigma_{2}(Z)= & 2,19-0,186 Z
\end{aligned}
$$

dargestellt werden. Die in Tab. 4 angegebenen $C_{2 i}$ gehören zum 2s-Einteilchen-Zustand. Zur Vollstän- 
digkeit geben wir noch die $C_{1 k}(k=1 \ldots 5)$ wieder, wenn die $(1 s)^{2}(2 s)^{2}$-Konfiguration vorliegt. Auch der 1s-Zustand wird jetzt mit den 5 Gauss-Funktionen, deren $\eta_{k}$ in (11) aufgeschrieben wurden, angenähert.

\begin{tabular}{|r|c|c|c|c|c|}
\hline$Z$ & $C_{11}$ & $C_{12}$ & $C_{13}$ & $C_{14}$ & $C_{15}$ \\
\hline $\mathbf{4}$ & 0,574 & 0,438 & 0,109 & 0,016 & 0,020 \\
5 & 0,582 & 0,432 & 0,103 & 0,015 & 0,024 \\
6 & 0,585 & 0,429 & 0,100 & 0,014 & 0,026 \\
7 & 0,587 & 0,428 & 0,098 & 0,014 & 0,027 \\
8 & 0,583 & 0,427 & 0,097 & 0,014 & 0,028 \\
9 & 0,587 & 0,427 & 0,096 & 0,014 & 0,028 \\
10 & 0,587 & 0,427 & 0,096 & 0,014 & 0,028 \\
\hline
\end{tabular}

Tab. 5.

Um für die in den nächsten Arbeiten folgenden Molekülrechnungen notwendigen $\eta_{k}{ }^{\prime}$ zu haben, sollen diese in den Tab. 6 und 7 angegeben werden:

\begin{tabular}{|r|c|c|c|c|c|}
\hline$Z$ & Atom/Ion & \multicolumn{1}{|c|}{$\eta_{1}^{\prime}$} & \multicolumn{1}{c|}{$\eta_{2}^{\prime}$} & \multicolumn{1}{c|}{$\eta_{3}^{\prime}$} & $\eta_{4}^{\prime}$ \\
\hline 2 & $\mathrm{He}$ & 0,297296 & 1,23414 & 6,05444 & 37,3059 \\
3 & $\mathrm{Li}^{+}$ & 0,813488 & 3,37697 & 16,5667 & 102,080 \\
4 & $\mathrm{Be}^{2+}$ & 1,58882 & 6,59555 & 32,3563 & 199,371 \\
5 & $\mathrm{~B}^{3+}$ & 2,62633 & 10,9025 & 53,5951 & 329,562 \\
6 & $\mathrm{C}^{4+}$ & 3,92847 & 16,3080 & 80,0034 & 492,961 \\
7 & $\mathrm{~N}^{5+}$ & 5,49725 & 22,8204 & 111,952 & 689,818 \\
8 & $\mathrm{O}^{6+}$ & 7,33432 & 30,4465 & 149,364 & 920,341 \\
9 & $\mathrm{~F}^{7+}$ & 9,44103 & 39,1918 & 192,266 & 1184,70 \\
10 & $\mathrm{Ne}^{8+}$ & 11,8185 & 49,0612 & 240,683 & 1483,03 \\
\hline
\end{tabular}

Tab. 6. $\eta_{k}{ }^{\prime}$-Werte für die Konfiguration $(1 \mathrm{~s})^{2}$.

\begin{tabular}{|r|c|c|c|c|c|c|}
\hline$Z$ & $\begin{array}{c}\text { Atom/ } \\
\text { Ion }\end{array}$ & $\eta_{1}^{\prime}$ & $\eta_{2}^{\prime}$ & $\eta_{3}^{\prime}$ & $\eta_{4}^{\prime}$ & $\eta_{5}^{\prime}$ \\
\hline 4 & $\mathrm{Be}$ & 1,76984 & 7,34701 & 36,0428 & 220,087 & 0,104367 \\
5 & $\mathrm{~B}^{+}$ & 2,95080 & 12,2495 & 60,0931 & $370,2790,223802$ \\
6 & $\mathrm{C}^{2+}$ & 4,41234 & 18,3166 & 89,8574 & $553,6790,388248$ \\
7 & $\mathrm{~N}^{3+}$ & 6,15367 & 25,5453 & 125,319 & 772,187 & 0,597704 \\
8 & $\mathrm{O}^{4+}$ & 8,17443 & 33,9339 & 166,472 & 1025,76 & 0,852173 \\
9 & $\mathrm{~F}^{5+}$ & 10,4744 & 43,4816 & 213,311 & 1314,37 & 1,15165 \\
10 & $\mathrm{Ne}^{6+}$ & 13,0545 & 54,1880 & 265,834 & 1638,00 & 1,49769 \\
\hline
\end{tabular}

Tab. 7. $\eta_{k}^{\prime}$-Werte für die Konfiguration $(1 \mathrm{~s})^{2}(2 \mathrm{~s})^{2}$.

Für Elektronenzahlen größer als vier bleibt in guter Näherung der Atomrumpf $(1 \mathrm{~s})^{2}(2 \mathrm{~s})^{2}$ erhalten, so daß dafür die fünf $\eta^{\prime}$-Werte nach Tab. 7 beibehalten werden können. Zur Beschreibung der 2pFunktionen verwenden wir jetzt die Darstellungen mit $M=2$ oder $M=4$ nach ( $6 \mathrm{a})$ und ( $6 \mathrm{~b})$. In diesem Falle tritt ein weiteres $\sigma$ auf ( $\sigma_{3}$ genannt), welches wieder variiert worden ist. Betrachten wir zuerst die Konfiguration $(1 s)^{2}(2 s)^{2}(2 p)^{2}$, so erhalten wir die Ergebnisse der Tab. 8, wenn wir vorerst nur den Ansatz (6 a) für $2 p$ verwenden.

Dazu sind vergleichsweise die entsprechenden HFErgebnisse aufgenommen worden.

\begin{tabular}{|r|l|l|l|l|l|l|}
\hline$Z$ & $\begin{array}{c}\text { Atom/ } \\
\text { Ion }\end{array}$ & $\sigma_{3}$ & $-\varepsilon_{\min }$ & $\begin{array}{c}-\varepsilon_{\mathrm{SCF}} \\
\text { nach }(9)\end{array}$ & $\Delta \varepsilon$ & $\%$ \\
\hline 6 & $\mathrm{C}$ & 3,42 & $+37,1167$ & $+37,68869$ & 0,5719 & 1,52 \\
7 & $\mathrm{~N}^{+}$ & 3,15 & $+52,9890$ & $+53,8900$ & 0,9010 & 1,67 \\
8 & $\mathrm{O}^{2+}$ & 3,17 & $+71,8408$ & $+73,1002$ & 1,2594 & 1,72 \\
9 & $\mathrm{~F}^{3+}$ & 3,13 & $+93,6431$ & $+95,3196$ & 1,6765 & 1,76 \\
10 & $\mathrm{Ne}^{4+}$ & 3,10 & $+118,3966$ & $+120,5435$ & 2,1469 & 1,78 \\
\hline
\end{tabular}

Tab. 8. Konfiguration $(1 s)^{2}(2 s)^{2}(2 p)^{2}$.

Verwenden wir zwei „GAuss-Funktionen-Pärchen“ nach $(6 \mathrm{~b})$, so resultieren die folgenden $\varepsilon$-Werte (Tab. 9).

\begin{tabular}{|r|l|c|c|c|c|c|}
\hline$Z$ & $\begin{array}{c}\text { Atom } \\
\text { Ion }\end{array}$ & $\sigma_{3}{ }^{\prime}$ & $-\varepsilon_{\min }$ & $\begin{array}{c}-\varepsilon_{\mathrm{SCF}} \\
\text { nach }(9)\end{array}$ & $\Delta \varepsilon$ & $\%$ \\
\hline 6 & $\mathrm{C}$ & 3,37 & $+37,4365$ & $+37,6886$ & 0,2521 & 0,67 \\
7 & $\mathrm{~N}^{+}$ & 3,32 & $+53,5293$ & $+53,8900$ & 0,3607 & 0,67 \\
8 & $\mathrm{O}^{2+}$ & 3,33 & $+72,6247$ & $+73,1002$ & 0,4755 & 0,65 \\
9 & $\mathrm{~F}^{3+}$ & 3,43 & $+94,7129$ & $+95,3196$ & 0,6067 & 0,64 \\
10 & $\mathrm{Ne}^{4+}$ & 2,96 & $+119,7989$ & $+120,5435$ & 0,7446 & 0,62 \\
\hline
\end{tabular}

Tab. 9. Konfiguration $(1 s)^{2}(2 s)^{2}(2 p)^{2}$.

Die Tabellierung in den $\sigma$ ergibt mehrere Energieminima, die dadurch hervorgerufen werden, daß für bestimmte $\sigma$-Werte der p-Funktionen, deren $\eta$-Werte mit einem $\eta$-Wert der fünf im Zentrum lokalisierten Gauss-Funktionen übereinstimmt. In diesem Falle ist das verwendete Funktionensystem nahezu linear abhängig, so daß die $\mathcal{E}$-Werte ansteigen. In den Tabellen sind nur die absoluten Minima aufgenommen worden! Die jeweiligen $\eta^{\prime}$.Werte und die Raumlagen

\begin{tabular}{|r|l|l|l||l|l|l|}
\hline$Z$ & $\begin{array}{c}\text { Atom } \\
\text { Ion }\end{array}$ & $\eta_{6}^{(1)}$ & $x_{6}^{(1)}$ & $\eta_{6}^{(2)}$ & $\eta_{8}^{(2)}$ & $x_{6.8}^{(2)}$ \\
\hline 6 & $\mathrm{C}$ & 0,3054 & $\pm 0,3619$ & 1,1672 & 0,2335 & $\pm 0,3042$ \\
7 & $\mathrm{~N}^{+}$ & 0,6817 & $\pm 0,2422$ & 2,2853 & 0,4571 & $\pm 0,2174$ \\
8 & $\mathrm{O}^{2+}$ & 1,0729 & $\pm 0,1931$ & 3,6802 & 0,7361 & $\pm 0,1713$ \\
9 & $\mathrm{~F}^{3+}$ & 1,5832 & $\pm 0,1589$ & 5,2354 & 1,0471 & $\pm 0,1436$ \\
10 & $\mathrm{Ne}^{4+}$ & 2,1895 & $\pm 0,1352$ & 8,3635 & 1,6727 & $\pm 0,1136$ \\
\hline
\end{tabular}

Tab. 10. Konfiguration $(1 s)^{2}(2 s)^{2}(2 p)^{2}$.

( $x$-Koordinaten) sind in Tab. 10 angegeben, wobei

$$
\begin{aligned}
& \eta_{6}{ }^{(1)^{\prime}} \equiv \eta_{7}{ }^{(1)^{\prime}}=0,0460\left(Z-\sigma_{3}{ }^{\prime}\right)^{2}, \\
& \eta_{6}{ }^{(2)^{\prime}} \equiv \eta_{7}{ }^{(2)^{\prime}}=0,1687\left(Z-\sigma_{3}{ }^{\prime}\right)^{2}, \\
& \eta_{8}{ }^{(2)^{\prime}} \equiv \eta_{9}{ }^{(2)^{\prime}}=0,0337\left(Z-\sigma_{3}{ }^{\prime}\right)^{2}
\end{aligned}
$$

und $x_{5}^{(1)}$ bzw. $x_{5,8}^{(2)}$ die Lagen der GAuss-Funktionen auf der $x$-Achse bedeuten, wenn 1 bzw. 2 "Pärchen“ verwendet werden. Die folgenden Tabellen enthalten eine Zusammenfassung der $C_{j k}$, wenn die Konfiguration $(1 s)^{2}(2 s)^{2}(2 p)^{2}$ vorliegt, wobei entweder ein (Tab. 11) oder zwei (Tab. 12) Gauss-Funktionenpaare für $2 p$ herangezogen wurden. 


\begin{tabular}{|c|c|c|c|c|c|c|c|c|}
\hline$Z / k$ & 1 & 2 & 3 & 4 & 5 & 6 & 7 & $j$ \\
\hline 6 & $\begin{array}{c}+0,5784 \\
-0,2106 \\
0\end{array}$ & $\begin{array}{c}+0,4300 \\
-0,1152 \\
0\end{array}$ & $\begin{array}{c}+0,1001 \\
-0,0218 \\
0\end{array}$ & $\begin{array}{c}+0,0142 \\
-0,0032 \\
0\end{array}$ & $\begin{array}{c}+0,1181 \\
+0,3445 \\
0\end{array}$ & $\begin{array}{r}-0,0457 \\
+0,3596 \\
+2,5502\end{array}$ & $\begin{array}{r}-0,0457 \\
+0,3596 \\
-2,5502\end{array}$ & 1 \\
\hline 7 & $\begin{array}{c}+0,5777 \\
-0,2343 \\
0\end{array}$ & $\begin{array}{c}+0,4290 \\
-0,1267 \\
0\end{array}$ & $\begin{array}{c}+0,0981 \\
-0,0236 \\
0\end{array}$ & $\begin{array}{c}+0,0139 \\
-0,0034 \\
0\end{array}$ & $\begin{array}{c}-0,1936 \\
+2,2248 \\
0\end{array}$ & $\begin{array}{l}+0,1156 \\
-0,5985 \\
+2,5502\end{array}$ & $\begin{array}{l}+0,1156 \\
-0,5985 \\
-2,5502\end{array}$ & l \\
\hline 8 & $\begin{array}{c}+0,5766 \\
-0,2462 \\
0\end{array}$ & $\begin{array}{c}+0,4287 \\
-0,1350 \\
0\end{array}$ & $\begin{array}{c}+0,0968 \\
-0,0246 \\
0\end{array}$ & $\begin{array}{c}+0,0138 \\
-0,0036 \\
0\end{array}$ & $\begin{array}{c}-0,0917 \\
+1,6164 \\
0\end{array}$ & $\begin{array}{r}+0,0648 \\
-0,2856 \\
+2,5501\end{array}$ & $\begin{array}{r}+0,0648 \\
-0,2856 \\
-2,5501\end{array}$ & 1 \\
\hline 9 & $\begin{array}{c}+0,5760 \\
-0,2579 \\
0\end{array}$ & $\begin{array}{c}+0,4286 \\
-0,1420 \\
0\end{array}$ & $\begin{array}{c}+0,0958 \\
-0,0255 \\
0\end{array}$ & $\begin{array}{c}+0,0136 \\
-0,0037 \\
0\end{array}$ & $\begin{array}{c}-0,0510 \\
+1,4003 \\
0\end{array}$ & $\begin{array}{l}+0,0447 \\
-0,1724 \\
+2,5501\end{array}$ & $\begin{array}{l}+0,0447 \\
-0,1724 \\
-2,5501\end{array}$ & 1 \\
\hline 10 & $\begin{array}{c}+0,5758 \\
-0,2678 \\
0\end{array}$ & $\begin{array}{c}+0,4286 \\
-0,1477 \\
0\end{array}$ & $\begin{array}{c}+0,0952 \\
-0,0263 \\
0\end{array}$ & $\begin{array}{c}+0,0136 \\
-0,0038 \\
0\end{array}$ & $\begin{array}{c}-0,0326 \\
+1,3142 \\
0\end{array}$ & $\begin{array}{r}+0,0356 \\
-0,1257 \\
+2,5502\end{array}$ & $\begin{array}{l}+0,0356 \\
-0,1257 \\
-2,5502\end{array}$ & 1 \\
\hline
\end{tabular}

Tab. 11. Die $C_{j k}$ bei $(1 \mathrm{~s})^{2}(2 \mathrm{~s})^{2}(2 \mathrm{p})^{2}$ mit einem Gauss-Funktionenpaar.

\begin{tabular}{|c|c|c|c|c|c|c|c|c|c|c|}
\hline$Z / k$ & 1 & 2 & 3 & 4 & 5 & 6 & 7 & 8 & 9 \\
\hline \multirow{2}{*}{6} & $+0,5670$ & $+0,4315$ & $+0,0996$ & $+0,0142$ & $+0,0188$ & $+0,0182$ & $+0,0182$ & $-0,0061$ & $-0,0061$ & 1 \\
& $-0,2450$ & $-0,1080$ & $-0,0226$ & $-0,0031$ & $+0,2318$ & $+0,0787$ & $+0,0787$ & $+0,3658$ & $+0,3658$ & 2 \\
& 0 & 0 & 0 & 0 & 0 & $+0,6327$ & $-0,6327$ & $+2,5612$ & $-2,5612$ & 3 \\
\multirow{2}{*}{7} & $+0,5723$ & $+0,4290$ & $+0,0979$ & $+0,0139$ & $+0,0903$ & $+0,0073$ & $+0,0073$ & $-0,0329$ & $-0,0329$ & 1 \\
& $-0,2792$ & $-0,1170$ & $-0,0243$ & $-0,1005$ & $-0,0033$ & $+0,0711$ & $+0,0711$ & $+0,5455$ & $+0,5455$ & 2 \\
& 0 & 0 & 0 & 0 & 0 & $+0,6089$ & $-0,6089$ & $+2,6023$ & $-2,6023$ & 3 \\
& $+0,5802$ & $+0,4269$ & $+0,0968$ & $+0,0137$ & $+0,1922$ & $-0,0008$ & $-0,0008$ & $-0,0795$ & $-0,0795$ & 1 \\
8 & $-0,3080$ & $-0,1247$ & $-0,256$ & $-0,0035$ & $-0,6197$ & $+0,0715$ & $+0,0715$ & $+0,8149$ & $+0,8149$ & 2 \\
& 0 & 0 & 0 & 0 & 0 & $+0,5977$ & $-0,5977$ & $+2,6213$ & $-2,6213$ & 3 \\
& $+0,5872$ & $+0,4257$ & $+0,0961$ & $+0,0136$ & $+0,2921$ & $-0,0062$ & $-0,0062$ & $-0,1276$ & $-0,1276$ & 1 \\
9 & $-0,3300$ & $-0,1312$ & $-0,0267$ & $-0,0036$ & $-1,0803$ & $+0,0731$ & $+0,0731$ & $+1,0529$ & $+1,0529$ & 2 \\
& 0 & 0 & 0 & 0 & 0 & $+0,6036$ & $-0,6036$ & $+2,6113$ & $-2,6113$ & 3 \\
& $+0,6164$ & $+0,4222$ & $+0,0959$ & $+0,0134$ & $-0,3558$ & $-0,0259$ & $-0,0259$ & $+0,2050$ & $+0,2050$ & 1 \\
10 & $-0,3804$ & $-0,1350$ & $-0,0276$ & $-0,0037$ & $+3,0277$ & $+0,0876$ & $+0,0876$ & $-0,1151$ & $-1,0151$ & 2 \\
& 0 & 0 & 0 & 0 & 0 & $+0,5174$ & $-0,5174$ & $+2,7536$ & $-2,7536$ & 3 \\
\hline
\end{tabular}

Tab. 12. Die $C_{j k}$ bei $(1 \mathrm{~s})^{2}(2 \mathrm{~s})^{2}(2 \mathrm{p})^{2}$ mit zwei Gauss-Funktionenpaaren.

Die $j$-Werte 1, 2 und 3 entsprechen den Einteilchenzuständen $1 \mathrm{~s}, 2 \mathrm{~s}$ und $2 \mathrm{p}$. Die Numerierung in $k$ ist durch die Ansätze [Erweiterungen von (9)]

$\psi_{j}=C_{j 1} \varphi_{1}+C_{j 2} \varphi_{2}+C_{j 3} \varphi_{3}+C_{j 4} \varphi_{4}+C_{j 5} \varphi_{5}$

$$
\underbrace{+C_{j 6} \varphi_{6}+C_{j 7} \varphi_{7}}_{\begin{array}{c}
\mathrm{p}_{x} \\
\text { (ein GaUss-Funktionenpaar) }
\end{array}}
$$

und

$$
\begin{gathered}
\psi_{j}=C_{j 1} \varphi_{1}+C_{j 2} \varphi_{2}+C_{j 3} \varphi_{3}+C_{j 4} \varphi_{4}+C_{j 5} \varphi_{5} \\
\underbrace{+C_{j 6} \varphi_{6}+C_{j 7} \varphi_{7}}_{\mathrm{p}_{x}} \underbrace{+C_{j 8} \varphi_{8}+C_{j 9} \varphi_{9}}_{\mathrm{p}_{x}} \\
\underbrace{}_{\text {(zwei Gauss-Funktionenpaare) }}
\end{gathered}
$$

festgelegt. Dabei ergeben sich im reinen $2 \mathrm{p}$-Fall für die Koeffizienten die Beziehungen

$$
C_{j 6}=-C_{j 7}
$$

bzw. $\quad C_{j 6}=-C_{j 7}$ und $C_{j 8}=-C_{j 9}$,

oder in bildlicher Darstellung Abb. 4 und Abb. 5 wie auch nach $(6 a)$ und $(6 \mathrm{~b})$ zu erwarten ist.

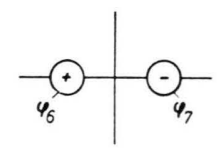

Abb. 4.

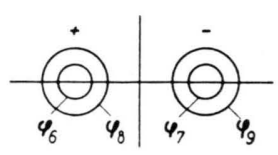

Abb. 5.
Für die Konfiguration $(1 s)^{2}(2 s)^{2}(2 p)^{4}$ ist in Erweiterung von (14) wie folgt anzusetzen:

$$
\begin{gathered}
\psi_{j}=C_{j 1} \varphi_{1}+C_{j 2} \varphi_{2}+C_{j 3} \varphi_{3}+C_{j 4} \varphi_{4}+C_{j 5} \varphi_{5} \\
+\underbrace{+C_{j 6} \varphi_{6}+C_{j 7} \varphi_{7}}_{\mathrm{p}_{x}}+\underbrace{C_{j 8} \varphi_{8}+C_{j 9} \varphi_{9}}_{\mathrm{p}_{y}} .
\end{gathered}
$$


In der folgenden Tab. 13 sind die Energien sowie die dazugehörenden variierten $\sigma_{4}$-Werte angegeben worden.

\begin{tabular}{|r|l|c|c|c|c|c|}
\hline$Z$ & $\begin{array}{c}\text { Atom } \\
\text { Ion }\end{array}$ & $\sigma_{4}$ & $-\varepsilon_{\min }$ & $\begin{array}{c}-\varepsilon_{\mathrm{SCF}} \\
\text { nach }(9)\end{array}$ & $\Delta \varepsilon$ & $\%$ \\
\hline 8 & $\mathrm{O}$ & 4,09 & $+72,7401$ & $+74,8094$ & 2,0693 & 2,77 \\
9 & $\mathrm{~F}^{+}$ & 4,10 & $+95,9941$ & $+98,8316$ & 2,8375 & 2,87 \\
10 & $\mathrm{Ne}^{2+}$ & 3,90 & $+122,6865$ & $+126,3719$ & 3,6854 & 2,92 \\
\hline
\end{tabular}

Tab. 13. Konfiguration $(1 s)^{2}(2 s)^{2}(2 p)^{4}$.

Verwendet man zwei „Gauss-Funktionen-Pärchen“, so ist anzusetzen

$$
\begin{gathered}
\psi_{j}=C_{j 1} \varphi_{1}+C_{j 2} \varphi_{2}+C_{j 3} \varphi_{3}+C_{j 4} \varphi_{4}+C_{j 5} \varphi_{5} \\
+\underbrace{C_{j 6} \varphi_{6}+C_{j 7} \varphi_{7}}_{\mathrm{p}_{x}}+\underbrace{C_{j 8} \varphi_{8}+C_{j 9} \varphi_{9}}_{\mathrm{p}_{x}} \\
\underbrace{+C_{j 10} \varphi_{10}+C_{j 11} \varphi_{11}}_{\mathrm{p}_{y}}+\underbrace{+C_{j 12} \varphi_{12}+C_{j 13} \varphi_{13}}_{\mathrm{p}_{y}}
\end{gathered}
$$

Tab. 14 gibt die Energiewerte sowie die variierten Werte des Parameters $\sigma_{4}{ }^{\prime}$ wieder, der, an Stelle von $\sigma_{4}$, im Falle zweier GAuss-Funktionenpaare auftritt.

\begin{tabular}{|r|l|c|c|c|c|c|}
\hline$Z$ & $\begin{array}{l}\text { Atom/ } \\
\text { Ion }\end{array}$ & $\sigma_{14}$ & $-\varepsilon_{\min }$ & $\begin{array}{c}-\varepsilon_{\mathrm{SCF}} \\
\text { nach }(9)\end{array}$ & $\Delta \varepsilon$ & $\%$ \\
\cline { 1 - 7 } 8 & $\mathrm{O}$ & 4,09 & $+73,1148$ & $+74,8094$ & 0,6946 & 0,93 \\
9 & $\mathrm{~F}^{+}$ & $\begin{array}{l}4,08 \\
+\quad 97,9619\end{array}$ & $+98,8316$ & 0,8697 & 0,88 \\
10 & $\mathrm{Ne}^{2+}$ & 4,06 & $+125,3072$ & $+126,3719$ & 1,0647 & 0,84 \\
\hline
\end{tabular}

Tab. 14. Konfiguration $(1 s)^{2}(2 s)^{2}(2 p)^{4}$.

\begin{tabular}{|r|l|c|c|c|c|c|}
\hline$Z$ & $\begin{array}{c}\text { Atom/ } \\
\text { Ion }\end{array}$ & $\eta_{6}^{(1)}$ & \multicolumn{1}{|c|}{$x_{6}^{(1)}$} & $\eta_{6}^{(2)}$ & $\eta_{8}^{(2)}$ & $x_{6,8}^{(2)}$ \\
\hline 8 & 0 & 0,7031 & $\pm 0,2385$ & 2,5799 & 0,5160 & $\pm 0,2046$ \\
9 & $\mathrm{~F}^{+}$ & 1,1042 & $\pm 0,1903$ & 4,0848 & 0,8170 & $\pm 0,1626$ \\
10 & $\mathrm{Ne}^{2+}$ & 1,7113 & $\pm 0,1529$ & 5,9541 & 1,1908 & $\pm 0,1347$ \\
\hline
\end{tabular}

Tab. 15. Konfiguration $(1 s)^{2}(2 s)^{2}(2 p)^{4}$.
Die Lage der Gauss-Funktionen sowie die $\eta^{\prime}$-Werte sind in Tab. 15 zusammengestellt, wobei $\eta_{6}^{(1)^{\prime}} \equiv \eta_{7}^{(1)^{\prime}} \equiv \eta_{12}^{(1)^{\prime}} \equiv \eta_{1}^{(1)^{\prime}}=0,0460\left(Z-\sigma_{4}\right)^{2}$ : $\eta_{6}^{(2)^{\prime}} \equiv \eta_{7}^{(2)^{\prime}} \equiv \eta_{10}^{(2)^{\prime}} \equiv \eta_{11}^{(2)^{\prime}}=0,1687\left(Z-\sigma_{4}{ }^{\prime}\right)^{2},(20)$ $\eta_{8}^{(2)^{\prime}} \equiv \eta_{9}^{(2)^{\prime}} \equiv \eta_{12}^{(2)^{\prime}} \equiv \eta_{13}^{(2)^{\prime}}=0,0337\left(Z-\sigma_{4}{ }^{\prime}\right)^{2}$.

Die Koeffizienten $C_{j k}$ sind in den Tab. 16 (ein Gauss-Funktionenpaar) und 17 (zwei Paare) zusammengefaßt.

Für den Fall der Konfiguration $(1 s)^{2}(2 s)^{2}(2 p)^{6}$ wurde das Ne-Atom berechnet. Wurden für jede pFunktion zwei Gauss-Funktionen verwendet, deren $\eta^{\prime}$ sich zu 1,2483 ergaben und deren Lagen $x^{(1)}=$ $\pm 0,1790$ betrugen, so resultierte im Minimum eine Gesamtenergie von $-123,5956$ at. E. Hier lautet der Ansatz

$$
\begin{gathered}
\psi_{j}=C_{j 1} \varphi_{1}+C_{j 2} \varphi_{2}+C_{j 3} \varphi_{3}+C_{j 4} \varphi_{4}+C_{j 5} \varphi_{5} \\
\underbrace{+C_{j 6} \varphi_{6}+C_{j 7} \varphi_{7}}_{\mathrm{p}_{x}} \underbrace{+C_{j 8} \varphi_{8}+C_{j 9} \varphi_{9}}_{\mathrm{p}_{y}} \\
+\underbrace{C_{j 10} \varphi_{10}+C_{j 11} \varphi_{11}}_{\mathrm{p}_{z}} .
\end{gathered}
$$

Die Koeffizienten sind in Tab. 18 angegeben. Wurden zwei Gauss-Funktionenpaare pro p-Funktion angesetzt, so ergab sich eine Gesamtenergie von $-127,1680$ at. E., wobei $x^{(2)}= \pm 0,1556$. Die beiden $\eta^{\prime}$-Werte betragen dabei 4,4583 und 0,8917.

Tab. 19 gibt die Koeffizienten wieder, wenn fol. gender Ansatz vorliegt:

$$
\begin{aligned}
\psi_{j}= & C_{j 1} \varphi_{1}+C_{j 2} \varphi_{2}+C_{j 3} \varphi_{3}+C_{j 4} \varphi_{4}+C_{j 5} \varphi_{5} \\
& +\underbrace{C_{j 6} \varphi_{6}+C_{j 7} \varphi_{7}}_{\mathrm{p}_{x}}+\underbrace{C_{j 8} \varphi_{8}+C_{j 9} \varphi_{9}}_{\mathrm{p}_{x}} \\
& +\underbrace{C_{j 10} \varphi_{10}+C_{j 11} \varphi_{11}}_{\mathrm{p}_{y}}+\underbrace{C_{j 12} \varphi_{12}+C_{j 13} \varphi_{13}}_{\mathrm{p}_{y}}
\end{aligned}
$$

\begin{tabular}{|c|c|c|c|c|c|c|c|c|c|c|}
\hline$Z / k$ & 1 & 2 & 3 & 4 & 5 & 6 & 7 & 8 & 9 & $j$ \\
\hline \multirow{3}{*}{8} & $+0,5794$ & $+0,4284$ & $+0,0969$ & $+0,0138$ & $+0,1349$ & $-0,0263$ & $-0,0263$ & $-0,0263$ & $-0,0263$ & 1 \\
& $-0,2215$ & $-0,1261$ & $-0,0226$ & $-0,0033$ & $-0,1518$ & $+0,3065$ & $+0,3065$ & $+0,02065$ & $+0,3065$ & 2 \\
& 0 & 0 & 0 & 0 & 0 & $-2,5502$ & $+2,5502$ & $+0,0061$ & $-0,0061$ & 3 \\
& 0 & 0 & 0 & 0 & 0 & $-0,0061$ & $+0,0061$ & $-2,5502$ & $+2,5502$ & 4 \\
& $+0,5791$ & $+0,4282$ & $+0,0960$ & $+0,0136$ & $+0,3367$ & $-0,0773$ & $-0,0773$ & $-0,0773$ & $-0,0773$ & 1 \\
9 & $-0,2367$ & $-0,1739$ & $-0,0238$ & $-0,0035$ & $-2,0704$ & $+0,7965$ & $+0,7965$ & $+0,7965$ & $+0,7965$ & 2 \\
& 0 & 0 & 0 & 0 & 0 & $-2,5402$ & $+2,5402$ & $+0,247$ & $-0,2247$ & 3 \\
& 0 & 0 & 0 & 0 & 0 & $-0,2247$ & $+0,2247$ & $-2,5402$ & $+2,5402$ & 4 \\
& $+0,5774$ & $+0,4283$ & $+0,0932$ & $+0,0135$ & $-0,1762$ & $+0,0541$ & $+0,0541$ & $+0,0541$ & $+0,0541$ & 1 \\
10 & $-0,2440$ & $-0,1394$ & $-0,0246$ & $-0,0036$ & $+2,8974$ & $-0,4707$ & $-0,4707$ & $-0,4707$ & $-0,4707$ & 2 \\
& 0 & 0 & 0 & 0 & 0 & $-1,5846$ & $+1,5846$ & $-1,9981$ & $+1,9981$ & 3 \\
& 0 & 0 & 0 & 0 & 0 & $+1,9981$ & $-1,9981$ & $-1,5846$ & $+1,5846$ & 4 \\
\hline
\end{tabular}

Tab. 16. Die $C_{j k}$ bei $(1 \mathrm{~s})^{2}(2 \mathrm{~s})^{2}(2 \mathrm{p})^{4}$ mit einem GAuss-Funktionenpaar pro p-Funktion. 


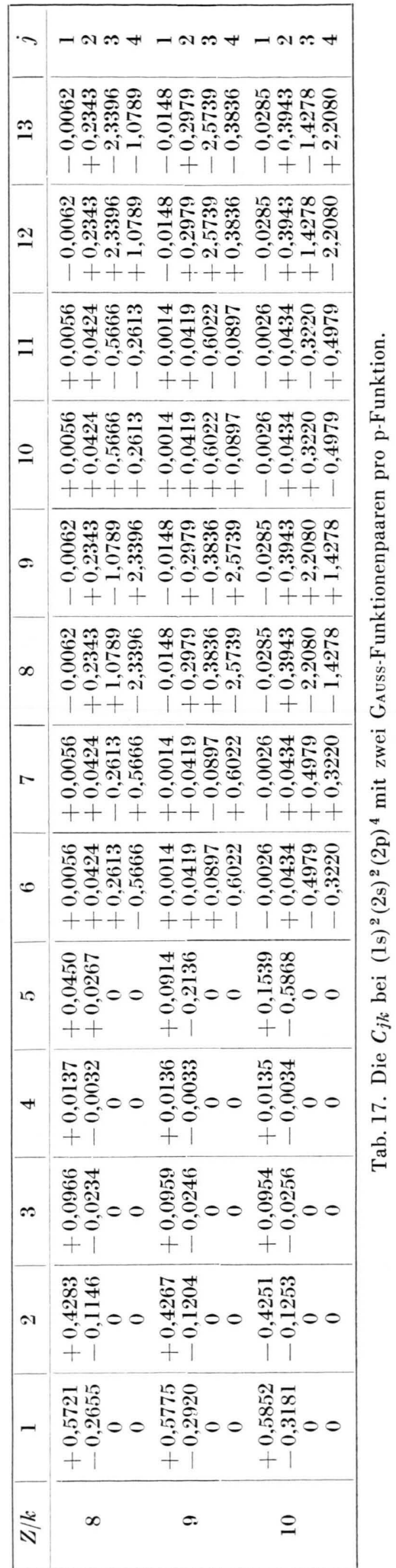

$$
+\underbrace{C_{j 14} \varphi_{14}+C_{j 15} \varphi_{15}}_{\mathrm{p}_{z}}+\underbrace{C_{j 16} \varphi_{16}+C_{j 17} \varphi_{17}}_{\mathrm{p}_{z}} .
$$

Der bisher beste SCF-Wert der Energie beträgt - 128,547 at. E. ${ }^{7}$.

Bei der Angabe der Koeffizienten ist noch zu bedenken, daß die Darstellung abgeschlossener Schalen, wie sie hier verwendet wird, noch willkürlich ist, bezüglich der Determinanteneigenschaft, daß Linearkombinationen der LCGO's (mit gleichen Spin), die wieder in die Determinante hineingeschrieben werden, die ursprüngliche Determinante um einen Zahlenfaktor verändern können, der aber wieder in die Normierung hineingenommen wird, so daß die Gesamtenergie dadurch nicht verändert wird. Auf diese Weise lassen sich aus den Koeffizientendarstellungen der Tab. $16-19$ reine s- und p-Anteile in den LCGO's herstellen.

\section{Diskussion der Ergebnisse}

Die Ergebnisse zeigen, daß zur Erfassung der Konfiguration $(1 s)^{2}(2 s)^{2}$, die in den meisten Atomen der ersten Achterperiode als Atomrumpf auftritt, fünf Gauss-Funktionen ausreichen, die im Zentrum des Atoms lokalisiert sind. Obwohl nicht alle $\eta^{\prime}$-Werte variiert wurden (was mit einer größeren Rechenmaschine ohne weiteres gemacht werden könnte), betragen die Abweichungen von den bekannten HF-Werten nur wenige Promille. Das gilt auch für die Mitberücksichtigung von p-Funktionen, wenn diese mit einem oder zwei Gauss-Funktionenpaaren approximiert werden.

Bemerkenswert ist die Tatsache, die sich aus den Tab. 2 und 5 ergibt, daß sich für $(1 \mathrm{~s})^{2}$ und (1s) ${ }^{2}$ $(2 \mathrm{~s})^{2}$ die Koeffizienten der LCGO-Ansätze nur wenig abhängig von der Kernladungszahl zeigen, was darauf hinweist, daß die Wahl der $\eta^{\prime}$-Werte nach (7), (8), (10), (11) und (12a,b) als Funktion der Kernladung vernünftig getroffen war, daß also besonders (7) die Verhältnisse gut beschreibt, was wir aus Betrachtungen der SLater-Funktionen und der dazugehörigen Schrödinger-Gleichung $(4 \mathrm{a}, \mathrm{b}, \mathrm{c})$ herauslasen.

Der Vergleich mit SCF-LCAO-Rechnungen für $(1 s)^{2}(2 s)^{2}$ zeigt $^{6,8}$, daß bei Verwendung von SLATER-Funktionen im LCAO-Ansatz ebenfalls erst von fünf Funktionen an der HF-Wert der Gesamtenergie

7 E. Clementi, J. Chem. Phys. 38, 996 [1963].

8 C. C. Roothana u. R. S. Kelly, Phys. Rev. 131, 1177 [1963]. 


\begin{tabular}{|l|c|c|c|c|c|c|c|c|c|c|c|}
\hline$j / k$ & 1 & 2 & 3 & 4 & 5 & 6 & 7 & 8 & 9 & 10 & 11 \\
\hline 1 & $+0,5793$ & $+0,4282$ & $+0,0954$ & $+0,0135$ & $+0,1329$ & $-0,0169$ & $-0,0169$ & $-0,0169$ & $-0,0169$ & $-0,0169$ & $-0,0169$ \\
2 & $-0,2260$ & $-0,1325$ & $-0,0231$ & $-0,0034$ & $-0,4340$ & $+0,2522$ & $+0,2522$ & $+0,2522$ & $+0,2522$ & $+0,2522$ & $+0,2522$ \\
3 & 0 & 0 & 0 & 0 & 0 & $-2,5215$ & $+2,5215$ & $-0,3226$ & $+0,3226$ & $-0,2023$ & $+0,2023$ \\
4 & 0 & 0 & 0 & 0 & 0 & $+0,2652$ & $-0,2652$ & $-2,4600$ & $+2,4600$ & $+0,6172$ & $-0,6172$ \\
5 & 0 & 0 & 0 & 0 & 0 & $+0,2733$ & $-0,2733$ & $-0,5893$ & $+0,5893$ & $-2,4660$ & $+2,4660$ \\
\hline
\end{tabular}

Tab. 18. Konfiguration (1s ${ }^{2}(2 s)^{2}(2 p)^{6}$ des Ne mit einem Gauss-Funktionenpaar pro p-Funktion.

\begin{tabular}{|l|c|c|c|c|c|c|c|c|}
\hline$j / k$ & 1 & 2 & 3 & 4 & 5 & 6 & 7 & 8 \\
\hline 1 & $+0,5758$ & $+0,4269$ & $+0,0952$ & $+0,0135$ & $+0,0590$ & $+0,0020$ & $+0,0020$ & $-0,0051$ \\
2 & $-0,2778$ & $-0,1185$ & $-0,0239$ & $-0,0032$ & $-0,0661$ & $+0,0306$ & $+0,0306$ & $+0,1707$ \\
3 & 0 & 0 & 0 & 0 & 0 & $+0,0882$ & $-0,0882$ & $+0,3646$ \\
4 & 0 & 0 & 0 & 0 & 0 & $-0,5951$ & $+0,5951$ & $-2,4589$ \\
5 & 0 & 0 & 0 & 0 & 0 & $+0,1644$ & $-0,1644$ & $+0,6793$ \\
\hline
\end{tabular}

\begin{tabular}{|c|c|c|c|c|c|c|c|c|c|}
\hline$j / k$ & 9 & 10 & 11 & 12 & 13 & 14 & 15 & 16 & 17 \\
\hline $\mathbf{1}$ & $-0,0051$ & $+0,0020$ & $+0,0020$ & $-0,0041$ & $-0,0051$ & $+0,0020$ & $+0,0020$ & $-0,0051$ & $-0,0051$ \\
2 & $+0,1707$ & $+0,0306$ & $+0,0306$ & $+0,1707$ & $+0,1707$ & $+0,0306$ & $+0,0306$ & $+0,1707$ & $+0,1707$ \\
3 & $-0,3646$ & $-0,5745$ & +0.5745 & $-2,3738$ & $+2,3738$ & $-0,2261$ & $+0,2261$ & $-0,9341$ & $+0,9341$ \\
4 & $+2,4589$ & $-0,1400$ & $+0,1400$ & $-0,5783$ & $+0,5783$ & $+0,1234$ & $-0,1234$ & $+0,5099$ & $-0,5099$ \\
5 & $-0,6793$ & $-0,1983$ & $+0,1983$ & $-0,8192$ & $+0,8192$ & $+0,5680$ & $-0,5680$ & $+2,3469$ & $-2,3469$ \\
\hline
\end{tabular}

Tab. 19. Konfiguration $(1 \mathrm{~s})^{2}(2 \mathrm{~s})^{2}(2 \mathrm{p})^{6}$ des Ne mit zwei GAuss-Funktionenpaaren pro p-Funktion.

in sehr guter Näherung erreicht wurde. Die Verwendung von 20 SLATER-Funktionen verbesserte die Energiewerte nur sehr unwesentlich. Daraus kann der Schluß gezogen werden, daß die Gauss-Funktionen in der hier verwendeten Form einen brauchbaren Funktionensatz darstellen, und da $\beta$ nicht befürchtet zu werden braucht, daß deren Anzahl im LCGO-Ansatz sehr groß werden muß, wenn die Güte der SCF-Rechnungen mit SLATER-Funktionen erreicht werden soll.

Dabei ist zu bedenken, daß in Molekülen die pFunktionen verändert sind und daher der Ansatz mit Gauss-Funktionen, wie er hier verwendet wird, elastischer ist, um auch Dichten zu erfassen, die zwischen den Zentren liegen (man vergleiche die Rechnungen am $\mathrm{H}_{3}{ }^{+}$). Es ist daher nicht notwendig, in Molekülen p-Funktionen zu verwenden, die diese Funktionen besonders gut in den freien Atomen darstellen. Die gegenseitigen Wechselwirkungen der Atome verändern die p-Funktionen sehr, dagegen scheinen nach den bisherigen Erfahrungen die (1s) $^{2}(2 s)^{2}$-Rümpfe weitgehend erhalten zu bleiben. In diesem Zusammenhang ist es wichtig, darauf hinzuweisen, daß vier reine Gauss-Funktionen in einem Zentrum eine größere Variabilität besitzen als eine entsprechende s-Funktion, die nur in der effektiven Kernladungszahl variiert werden kann.
Mit den vorliegenden Ergebnissen ist nun der Weg zu Molekülrechnungen mit Hilfe des SCF. LCGO-Verfahrens frei, wenn die Moleküle Atome der ersten Achterperiode enthalten und eine geradzahlige Anzahl von Elektronen erhalten, weil im Rahmen des Verfahrens mit der Darstellung der "geschlossenen Schalen“ gearbeitet wird.

Der nächste Schritt kann daher in einer Anwendung des CI-Verfahrens bestehen, wobei dort LCGO-Funktionen verwendet werden, die sich in der SCF-LCGO-Methode ergaben. Es handelt sich also dann um ein CI-Verfahren mit reinen GAussFunktionen (KGO-Verfahren $\left.{ }^{9}\right)$. In diesen Verfahren fällt ganz allgemein die Beschränkung auf geradzahlige Elektronensysteme weg, und die Berechnung von $\psi$ und $\varepsilon$ kann im Prinzip beliebig genau durchgeführt werden. Programme dieser Art mit LCGO-Funktionen sind in Vorbereitung. Die Bindungsenergien, die in diesem Rahmen berechnet werden, werden in der Regel auf den Übergang zu den getrennten Atomen innerhalb des Verfahrens bezogen, so da $ß$ damit erfahrungsgemäß einige der Ungenauigkeiten der Energierechnungen bei den Molekülen und Atomen herausfallen. Das CI-Verfahren liefert auch angeregte Zustände ${ }^{9}$.

9 H. Preuss, Mol. Phys. 8, 157 [1964]. 
Die Erweiterungen auf Atome mit Kernladungen größer als zehn kann in entsprechender Weise, wie hier angegeben, vorgenommen werden. So wäre eine 3s-Funktion mit einem LCGO-Ansatz mit sechs oder sieben Gauss-Funktionen zu approximieren, wobei die ersten fünf (oder sechs) von den vorherigen Rechnungen zu übernehmen wären. Gegebenenfalls könnten auch mehr Funktionen im Zen- trum lokalisiert werden. Während die Ansätze für p-Funktionen erhalten bleiben, wäre für d-Funktionen etwa wie in Abb. 3 vorzugehen.

Frau I. Funke danke ich herzlich für die Herstellung der Programme an der Rechenmaschine G 3 und für die Durchführung der umfangreichen Rechnungen. Dem Verband der Chemischen Industrie möchte ich für die Bereitstellung von Mitteln für die wissenschaftlichen Untersuchungen meinen Dank sagen.

\title{
Uber den Einfluß von Fallen auf die Diffusion
}

\author{
(Edelgasdiffusion in Festkörpern. 24. Mitteilung \\ H. Gaus \\ Hahn-Meitner-Institut für Kernforschung Berlin \\ (Z. Naturforschg. 20 a, 1298-1308 [1965] ; eingegangen am 30. Juni 1965)
}

\begin{abstract}
It is generally assumed, that in solids the diffusion of gas is modified through traps, which capture the gas atoms for a certain length of time. Taking into account this trapping with constant absorption- and emission-probability the diffusion from a sphere was calculated numerically by Hurst. In the present paper the calculation is performed analytically for small diffusion depth (arbitrary body form). For the resulting gas release simple approximation formulas are obtained valid for different time intervals. With these formulas one may get values of certain parameter combinations directly from the experimental curves. The meaning of the absorption and emission probability is discussed using the theory of EYRING, ZENER and others. Especially for the quotient of absorption and emission probability a simple expression is obtained. In connection with the above mentioned release formulas this expression may explain the twofold activation enthalpy recently found by Richter and Zrmen.
\end{abstract}

Bei Messungen der Diffusion von Gasen aus Festkörpern wird häufig eine Abweichung von dem einfacher Volumendiffusion entsprechenden Verhalten gefunden, das sich durch die Annahme sogenannter Fallen (traps) im Festkörper erklären läßt ${ }^{1-4}$. Diese Fallen sollen die Gasatome einfangen und zumindest eine Zeit lang an der Diffusion hindern. Als derartige Fallen könnten Poren wirken, ferner räumlich begrenzte Versetzungslinien und andere Arten von Fehlstellen, insbesondere durch Bestrahlung hervorgerufene. Im folgenden soll der Einfluß dieser Fallen auf die Zeitabhängigkeit der Gasabgabe, also die sogenannte Kinetik des Diffusionsprozesses, und ihre Wirkungsweise im atomistischen Bild nach der von Eyring, Zener u. a. angegebenen Theorie untersucht werden.

Die Kinetik ist unter bestimmten Annahmen numerisch von Hurst ${ }^{1}$ berechnet worden. Er berück-

1 D. G. Hurst, AECL-1550, Nov. 1962.

2 J. R. MacEwan u. W. H. Stenens, J. Nucl. Mater. 11, 77 [1964].

3 F. Felix, P. Schmeling u. K. E. Zimen, EUR 259.d [1963]. sichtigt die Fallen durch einfache Absorptions- und Quellterme in der Diffusionsgleichung [siehe weiter unten Gl. (1) und (2) ]. Dies bedeutet, daß die Emissionswahrscheinlichkeit für ein eingefangenes und der Absorptionsquerschnitt für ein diffundierendes Atom sich zeitlich nicht ändern und unabhängig von der Besetzung der Fallen sind, und daß die Fallen räumlich gleichmäßig verteilt sind.

Die von Hurst berechneten Werte, die in Form von Tabellen und Kurven mitgeteilt werden, gelten für Kugel und zweiseitig begrenzte Platte. Wie Hurst bemerkt, ist jedoch für hinreichend geringe Gasabgabe, d. h. so lange die Diffusionstiefe klein gegen die Körperabmessungen ist, die prozentuale Gasabgabe wie bei regulärer Diffusion ${ }^{5}$ hinsichtlich der Form des Körpers nur durch das Verhältnis Oberfläche zu Volumen $(S / V)$ bestimmt. In Abschnitt 2 und 3 werden für diesen Bereich, der bei

${ }^{4}$ A. K. H. Richter u. K. E. Zimen, Z. Naturforschg. 20 a, 666 [1965].

5 W. Inthoff u. K. E. Zimen, Trans. Chalmers Univ. Technol. (Göteborg) No. 176, p. 16 [1956]. 